\title{
To hydrate or not to hydrate? The effect of hydration on survival, symptoms and quality of dying among terminally ill cancer patients
}

\author{
Chien-Yi Wu' ${ }^{1}$, Ping-Jen Chen ${ }^{1,2,3}$, Tzu-Lin Ho ${ }^{4}$, Wen-Yuan Lin ${ }^{5,6}$ and Shao-Yi Cheng ${ }^{4^{*}}$ (I)
}

\begin{abstract}
Background: Artificial nutrition and hydration do not prolong survival or improve clinical symptoms of terminally ill cancer patients. Nonetheless, little is known about the effect of artificial hydration (AH) alone on patients' survival, symptoms or quality of dying. This study explored the relationship between $\mathrm{AH}$ and survival, symptoms and quality of dying among terminally ill cancer patients.

Methods: A pilot prospective, observational study was conducted in the palliative care units of three tertiary hospitals in Taiwan between October 2016 and December 2017. A total of 100 patients were included and classified into the hydration and non-hydration group using $400 \mathrm{~mL}$ of fluid per day as the cut-off point. The quality of dying was measured by the Good Death Scale (GDS). Multivariate analyses using Cox's proportional hazards model were used to assess the survival status of patients, the Wilcoxon rank-sum test for within-group analyses and the Mann-Whitney $U$ test for between-groups analyses to evaluate changes in symptoms between day 0 and 7 in both groups. Logistic regression analysis was used to assess the predictors of a good death.

Results: There were no differences in survival $(p=0.337)$ or symptom improvement between the hydration and non-hydration group, however, patients with AH had higher GDS scores.

Conclusions: AH did not prolong survival nor significantly improve dehydration symptoms of terminally ill cancer patients but it may influence the quality of dying. Communication with patients and their families on the effect of AH may help them better prepared for the end-of-life experience.
\end{abstract}

Keywords: Artificial hydration, Cancer, Survival, Quality of dying, Palliative care

\footnotetext{
* Correspondence: scheng2140@gmail.com

${ }^{4}$ Department of Family Medicine, College of Medicine and University

Hospital, National Taiwan University, 7 Chung-Shan South Road, Taipei 100,

Taiwan

Full list of author information is available at the end of the article
}

C C The Author(s). 2021 Open Access This article is licensed under a Creative Commons Attribution 4.0 International License, which permits use, sharing, adaptation, distribution and reproduction in any medium or format, as long as you give appropriate credit to the original author(s) and the source, provide a link to the Creative Commons licence, and indicate if changes were made. The images or other third party material in this article are included in the article's Creative Commons licence, unless indicated otherwise in a credit line to the material. If material is not included in the article's Creative Commons licence and your intended use is not permitted by statutory regulation or exceeds the permitted use, you will need to obtain permission directly from the copyright holder. To view a copy of this licence, visit http://creativecommons.org/licenses/by/4.0/. The Creative Commons Public Domain Dedication waiver (http://creativecommons.org/publicdomain/zero/1.0/) applies to the data made available in this article, unless otherwise stated in a credit line to the data. 


\section{Background}

Previous studies have found that patients who receive palliative care have a better quality of life (QOL) as well as end-of-life experience [1-3]. In the clinical practice of end-of-life care, terminally ill cancer patients may cease to benefit from oral nutrition and fluids during the very terminal stage $[4,5]$. However, many family members and even patients themselves request medical staff to continuously administer artificial hydration (AH) [5-7]. Therefore, medical professionals often encounter an ethical dilemma related to the provision of artificial nutrition and hydration $(\mathrm{ANH})[8,9]$.

A Taiwanese study found that ANH did not prolong the survival of terminally ill cancer patients [6], and a randomised controlled trial of the influence of $\mathrm{AH}$ on terminally ill cancer patients showed no obvious difference in dehydration symptoms, QOL and survival between groups receiving $1 \mathrm{~L}$ and $100 \mathrm{ml}$ of fluid daily [10]. In a Japanese study, except for the improvement in membranous dehydration symptoms, hydration provided no benefit, but instead exacerbated fluid overload, induced hypoalbuminemia and failed to correct electrolyte imbalance [11-14]. Therefore, Japanese clinical guidelines do not suggest that medical professionals administer AH routinely if there is no specific need [15]. Indeed, the patient's condition, fluid overload condition and the attitude of family members are key factors in whether to administer $\mathrm{AH}$ [16]. In another Japanese study of over 5000 members of the general population and 800 bereaved family members, 33 to $50 \%$ of respondents believed that administering $\mathrm{AH}$ to terminally ill patients during the very terminal stage was a part of basic care, with 15 to $31 \%$ of respondents believing that $\mathrm{AH}$ could relieve symptoms [17]. In a western study, ethnicity played an important role in whether $\mathrm{AH}$ was perceived as food or medicine. Ethnic minorities in the United States, such as African Americans, Latinos and Asian Americans (total 66\%), were significantly more likely to view $\mathrm{AH}$ as food or as both food and medicine than non-Hispanic white subjects (42\%) [18]. In an Italian study, patients and their families considered $\mathrm{AH}$ as useful medical management, with most preferring the intravenous route, as they thought it could improve clinical conditions and had a positive psychological meaning [7]. Thus, cross-cultural comparison of the role of ANH is both practical and culturally sensitive.

Previous research shows that AH may be more harmful than beneficial to terminally ill cancer patients' QOL. However, little is known about the influence of $\mathrm{AH}$ on patients' quality of dying, therefore, the primary outcome of this pilot prospective observational study was to investigate the influence of $\mathrm{AH}$ on patients' quality of dying. Also, the relationship between $\mathrm{AH}$ and the survival and symptoms were assessed. It was hypothesised that $\mathrm{AH}$ would not affect the quality of dyig or improve dehydration symptoms or prolong the survival period.

\section{Methods \\ Study design and participants}

A pilot prospective, observational study was conducted in the palliative care units (PCU) of three tertiary hospitals in different cities in Taiwan (National Taiwan University Hospital, Chi-Mei Medical Centre and Kaohsiung Medical University Hospital) between October 2016 and December 2017. These hospitals were selected as they have abundant palliative care experience, as their PCU have been operational for more than 10 years, and they were willing to participate in the clinical observational study. This study was approved by Institutional Review Boards of all three hospitals.

The inclusion criteria for study objects were: (1) patients aged 20 years or older, (2) patients with locally advanced or metastatic cancer (histological, cytological or clinical diagnosis), (3) patients who could not have normal oral intake and (4) patients presenting with malaise and at least one of the following dehydration symptoms, delirium, dry mouth or myoclonus. The exclusion criteria were: (1) patients died less than $24 \mathrm{~h}$ after the admission to PCU, (2) patients or their family members declined participation and (3) patients with non-cancer terminal disease. All terminally ill patients in these three PCUs were screened for their eligibility during admission. If the patients met the inclusion criteria, the researchers explained the study purpose and protocol to the patients or their families (proxy) if patients had a conscious disturbance. The patients or their proxy provided written informed consent to participate in the study.

\section{Outcome measurements}

On admission to PCU, the need for $\mathrm{AH}$ by intravenous or subcutaneous route was according to clinical evaluation and management. After discussion with patients or their families about $\mathrm{AH}$, the duty physician administered the formulated $\mathrm{AH}$ to the patients as required. Patients were classified into the hydration group and the nonhydration group using $400 \mathrm{~mL}$ per day as the cut-off point, as the bottle of formulated $\mathrm{AH}$ which contains glucose and electrolytes is often $400 \mathrm{~mL}$ and is routinely administered to terminally ill cancer patients as a basic fluid supply. The daily hydration volume was calculated together with formulated $\mathrm{AH}$ and other fluids for medical purposes, such as antibiotics, albumin or blood transfusion. The two groups were compared to determine the effect of hydration on survival time, symptom relief, Good Death Scale (GDS) and the possible side effects of hydration. 
Other recorded variables included patient's age, gender, primary cancer, Charlson Comorbidity Index, social state, religion, clinical symptoms (including the eating condition by mouth, dyspnoea, fatigue, drowsiness, dry mouth, anorexia, muscle spasm, dysphagia, respiratory tract secretion, oedema, ascites, pleural effusion, bowel obstruction, water intake condition and delirium), blood transfusion, antibiotics use or albumin supply and patient's functional status as measured by the Eastern Cooperative Oncology Group performance status (ECOG). The eating condition by mouth was classified into reduced but more than a mouthful and less than a mouthful every time while eating. Dyspnoea was classified into no and yes, and the dyspnoea level was further divided into exertional only and at rest. The Integrated Palliative care Outcome Scale (IPOS) was developed to measure the patient's symptom severity. The ranking was: 0 , not at all; 1 , slightly; 2 , moderately; 3 , severely; 4 , overwhelmingly; 5, cannot assess. IPOS was used to assess the fatigue, drowsiness, dry mouth and anorexia symptoms. The myoclonus variable evaluated the patient's worst condition while at rest according to the ranking: 0 , none; $1, \leq 1$ jerk; $2,2-3$ jerks; $3,4-9$ jerks; and $4, \geq 10$ jerks per $10 \mathrm{~s}$. Dysphagia was divided into no or yes. The respiratory tract secretion variable evaluated the patient's worst condition, the scale was 0 , not audible; 1 , only audible at the head of the bed; 2 , clearly audible at the foot of the bed, and 3, clearly audible at $6 \mathrm{~m}$ from the foot of the bed. Lower extremity oedema was measured by observing the leg with less oedema and ranking 0 as none, 1 as mild $(<5 \mathrm{~mm}), 2$ as moderate $(5-10 \mathrm{~mm})$ and 3 as severe $(>10 \mathrm{~mm})$. Ascites and pleural effusion were evaluated by clinical examination or imaging, ranking 0 as none, 1 as physically detectable but asymptomatic and 2 as symptomatic. Bowel obstruction was classified into no or yes. The delirium level was evaluated using item 9 of the Memorial Delirium Assessment Scale (MDAS), decreased or increased psychomotor activity. The clinical symptoms were evaluated by the main healthcare professionals at baseline during admission to PCU and 1 week after enrollment until death.

\section{Good death scale (GDS)}

The GDS was used to evaluate the quality of dying [1921] according to five domains scored on a 4-point Likert scale: an awareness that one is dying $(0$, complete ignorance; 3 , complete awareness), acceptance of death peacefully $(0$, complete unacceptance; 3 , complete acceptance), honouring of the patient's wishes $(0$, no reference to the patient's wishes; 1 , following the family's wishes alone; 2, following the patient's wishes alone, and 3 , following the wishes of the patient and the family), death timing $(0$, no preparation; 1 , the family alone had prepared; 2 , the patient alone had prepared; and 3, both the patient and the family had prepared) and the degree of physical comfort 3 days before death $(0$, a lot of suffering; 1 , suffering; 2 , a little suffering; and 3 , no suffering). The GDS score, ranging from 0 to 15 , was discussed by the experienced palliative care team at the team meeting after each patient died. The score of each item was considered separately and the final score was decided by consensus at the team meeting. The higher the total score, the better the good death status the patient had achieved. The GDS of 68 patients were collected and analysed. A GDS $\geqq 12$ indicated a better quality of dying according to the quality indicator set at the National Taiwan University Hospital.

\section{Statistical analysis}

Descriptive analyses were used to assess the differences in demographic characteristics between the two groups. The Kaplan-Meier curve was used to estimate the impact of hydration on survival between the two groups and multivariate analyses using Cox's proportional hazards model were used to assess the survival time of patients. The Wilcoxon rank-sum test was applied for within-group analyses and the Mann-Whitney U test for between-groups analyses to evaluate changes in symptoms between day 0 and 7 in the hydration and nonhydration group. Finally, logistic regression analysis was used to assess the predictors for patients whose GDS $\geqq 12$. The $\mathrm{R}$ software was used for the statistical analyses (R Core Team, Foundation for Statistical Computing, Vienna, Austria) and a $p$-value $<0.05$ indicated statistical significance.

\section{Results}

A total of 133 patients were eligible for enrolment in this study, of which, 33 were excluded for the following reasons: 8 patients died within $24 \mathrm{~h}$ after admission, $7 \mathrm{pa}$ tients declined to participate, 13 patients had normal oral intake and 5 patients had a non-cancerous disease. Finally, 100 patients were analysed in this study, 22 in the hydration group and 78 in the non-hydration group. The patient recruitment flow chart is shown in Fig. 1, with the demographic and clinical characteristics of the enrolled patients provided in Table 1.

The average age of participants was $69.19 \pm 12.89$ years, with the non-hydration group being significantly older $(71.26 \pm 11.86$ years $)$ than the hydration group $(61.86 \pm$ 13.97 years) $(p=0.005)$. The mortality rate in hospital was significantly higher in the hydration group than the nonhydration group $(p=0.041)$. The non-hydration group had a better oral intake condition during admission than the hydration group $(p=0.008)$, and the groups also differed significantly with regards to religion $(p=0.015)$. There were no significant differences in hospital, gender, education level, cancer type, ECOG, marital status, bowel 


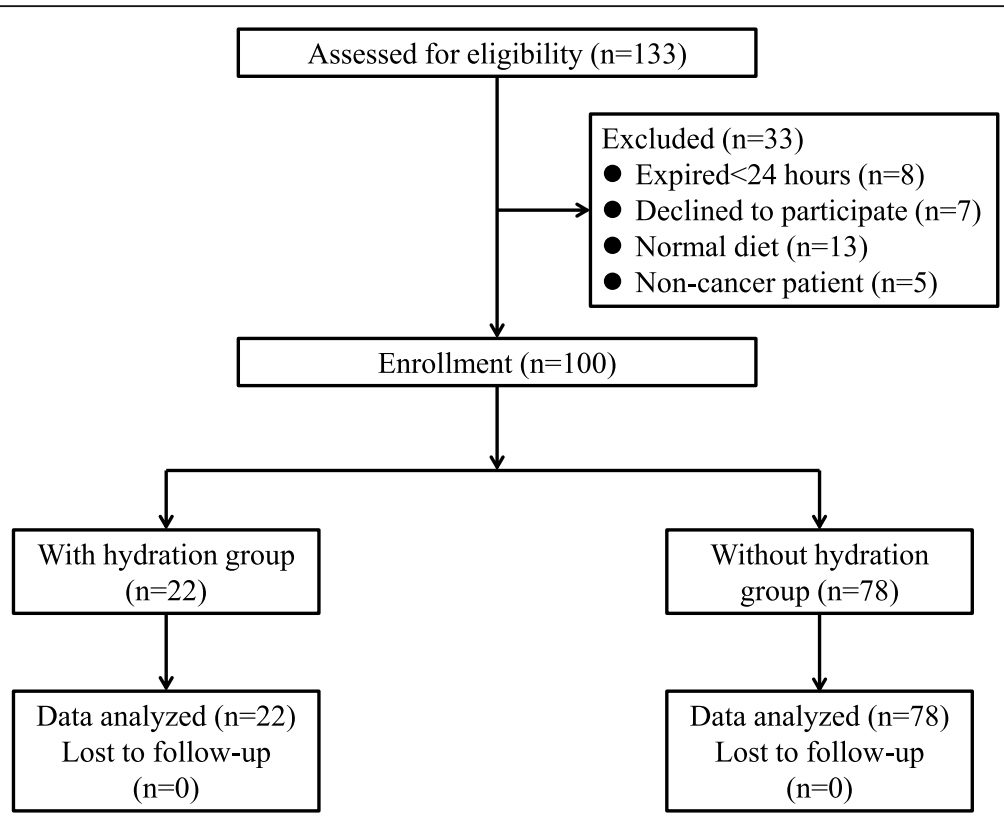

Fig. 1 The patient recruitment flow chart

obstruction, blood transfusion, antibiotics use or albumin use between the two groups $(p>0.05)$.

The survival analysis (Fig. 2) revealed no significance $(p=0.0552)$ difference in hospital survival time between the non-hydration group and the hydration group. Multivariate analyses of Cox's proportional hazards analysis of 68 deceased patients was applied to identify the prognostic factors related to mortality and the results are shown in Table 2. The risk of death was higher in those with unknown religion (HR: 9.844, 95\% CI: 1.42667.948) and fatigue or oedema during admission (HR: 1.722, 95\% CI: 1.072-2.767, and HR: 1.469 , 95\% CI: 1.068-2.019, respectively). The hospital, age, education level, oral intake status, artificial hydration amount, other physical symptoms and functional status during admission were not related to the risk of death.

The change in symptoms between day 0 and day 7 in these two groups are shown in Table 3, with no significant change in fatigue, dry mouth, myoclonus, delirium, dyspnoea or oedema. Regarding drowsiness symptoms, both the hydration and the non-hydration groups had more severe symptoms on day 7 than day $0(p=0.008$ and 0.038 , respectively), with the hydration group having a greater change in drowsiness than the non-hydration group $(p=0.019)$.

Sixty-eight patients died during hospitalisation in the PCUs and logistic regression was applied to analyse the predictors of a good death, as shown in Table 4. A GDS $\geqq 12$ indicates a better quality of dying for patients, with only hydration of $86-445 \mathrm{cc}$ significantly associated with a good death $(p=0.0011$, odds ratio [OR]: $12.8560,95 \%$ CI: 2.774-59.575).

\section{Discussion}

This study investigated the effect of $\mathrm{AH}$ on the survival period, symptom relief and quality of dying of terminally ill cancer patients, showing that the administration of $\mathrm{AH}$ did not prolong survival or improve dehydration symptoms but was associated with a better quality of dying for terminally ill cancer patients.

Morita et al. found that $\mathrm{AH}$ did not affect the presence of delirium in terminally ill cancer patients [11]. In a subsequent study, however, the administration of intravenous $\mathrm{AH}$ worsened fluid retention symptoms in terminal lung and gastric cancer patients. Reducing the volume of intravenous hydration improved fluid retention symptoms without any deterioration of dehydration symptoms [12]. In terminal patients with abdominal malignancies, patients given $1 \mathrm{~L}$ or more $\mathrm{AH}$ per day, although they had lower dehydration scores than those who received less than $1 \mathrm{~L} \mathrm{AH,} \mathrm{had} \mathrm{higher} \mathrm{symptom}$ scores for oedema, ascites and pleural effusion [13]. Nakajima et al. also reported that the symptom scores for oedema, ascites and bronchial secretion were higher in patients who received more than $1 \mathrm{~L}$ of $\mathrm{AH}$ per day [22], whereas Bruera et al. found no difference in dehydration symptoms, such as fatigue, myoclonus, drowsiness and delirium, 4 days later between patients who received $1 \mathrm{~L}$ or $100 \mathrm{ml}$ normal saline per day [10]. Our study showed no significant change in fatigue, delirium, dry mouth or myoclonus after 1 week between the hydration and non-hydration groups. Furthermore, the drowsiness level was more severe in the hydration group. In our study, we used $400 \mathrm{~mL}$ as the cut-off point to separate hydration or 
Table 1 Demographic and clinical characteristics of enrolled patients $(n=100)$

\begin{tabular}{|c|c|c|c|c|}
\hline \multirow[t]{2}{*}{ Variable } & \multirow[t]{2}{*}{ Total } & \multicolumn{2}{|l|}{ Hydration } & \multirow[t]{2}{*}{$p$-value } \\
\hline & & $\begin{array}{l}<400 \mathrm{ml} \\
(n=78)\end{array}$ & $\begin{array}{l}\geqq 400 \mathrm{ml} \\
(n=22)\end{array}$ & \\
\hline Institution & & & & 0.237 \\
\hline NTUH & 43 (43\%) & 37 (47.4\%) & $6(27.3 \%)$ & \\
\hline Chi-Mei & $26(26 \%)$ & 19 (24.4\%) & 7 (31.8\%) & \\
\hline $\mathrm{KMUH}$ & $31(31 \%)$ & $22(28.2 \%)$ & $9(40.9 \%)$ & \\
\hline Age & $69.19 \pm 12.89$ & $71.26 \pm 11.86$ & $61.86 \pm 13.97$ & 0.005 \\
\hline Gender & & & & 0.630 \\
\hline Female & $52(52 \%)$ & $42(53.8 \%)$ & $10(45.5 \%)$ & \\
\hline Male & 48 (48\%) & $36(46.2 \%)$ & $12(54.5 \%)$ & \\
\hline Education & & & & 0.053 \\
\hline$\leqq 6$ years & 49 (49\%) & $43(55.1 \%)$ & $6(27.3 \%)$ & \\
\hline $7 \sim 12$ years & 35 (35\%) & $23(29.5 \%)$ & $12(54.5 \%)$ & \\
\hline$>12$ years & $16(16 \%)$ & $12(15.4 \%)$ & $4(18.2 \%)$ & \\
\hline Marital status & & & & 0.190 \\
\hline Unmarried & $6(6.0 \%)$ & $5(6.4 \%)$ & $1(4.5 \%)$ & \\
\hline Married & $58(58.0 \%)$ & $44(56.4 \%)$ & $14(63.6 \%)$ & \\
\hline Widowed & $27(27.0 \%)$ & $24(30.8 \%)$ & $3(13.6 \%)$ & \\
\hline Separated / divorced & $9(9.0 \%)$ & $5(6.4 \%)$ & $4(18.2 \%)$ & \\
\hline Religion & & & & 0.015 \\
\hline Nullifidian & $11(11 \%)$ & $7(9.0 \%)$ & $4(18.2 \%)$ & \\
\hline Buddhism & $24(24 \%)$ & $23(29.5 \%)$ & $1(4.5 \%)$ & \\
\hline $\begin{array}{l}\text { Christian/ } \\
\text { Catholicism }\end{array}$ & $12(12 \%)$ & $12(15.4 \%)$ & $0(0 \%)$ & \\
\hline Taoism/Taiwanese folk religion & $50(50 \%)$ & $34(43.6 \%)$ & $16(72.7 \%)$ & \\
\hline Unknown & $3(3 \%)$ & $2(2.6 \%)$ & $1(4.5 \%)$ & \\
\hline Cancer & & & & 0.279 \\
\hline Lung & $15(15.0 \%)$ & $14(17.9 \%)$ & $1(4.5 \%)$ & \\
\hline Gl tract & $24(24.0 \%)$ & $16(20.5 \%)$ & $8(36.4 \%)$ & \\
\hline Liver/pancreas & $37(37.0 \%)$ & $29(37.2 \%)$ & $8(36.4 \%)$ & \\
\hline Breast & $3(3.0 \%)$ & $1(1.3 \%)$ & $2(9.1 \%)$ & \\
\hline Gynaecology & $6(6.0 \%)$ & $4(5.1 \%)$ & $2(9.1 \%)$ & \\
\hline Urinary tract & $8(8.0 \%)$ & $7(9.0 \%)$ & $1(4.5 \%)$ & \\
\hline Lymphoma & $1(1.0 \%)$ & $1(1.3 \%)$ & $0(0 \%)$ & \\
\hline Head and neck/brain & $3(3.0 \%)$ & $3(3.8 \%)$ & $0(0 \%)$ & \\
\hline Others & $3(3.0 \%)$ & $3(3.8 \%)$ & $0(0 \%)$ & \\
\hline ECOG & & & & 0.666 \\
\hline 1 & $1(1.0 \%)$ & $1(1.3 \%)$ & $0(0 \%)$ & \\
\hline 2 & $8(8.0 \%)$ & $5(6.4 \%)$ & $3(13.6 \%)$ & \\
\hline 3 & $43(43.0 \%)$ & $34(43.6 \%)$ & 9 (40.9\%) & \\
\hline 4 & $48(48.0 \%)$ & $38(48.7 \%)$ & $10(45.5 \%)$ & \\
\hline Oral intake & & & & 0.008 \\
\hline Less than a mouthful & $47(47.0 \%)$ & $31(39.7 \%)$ & $16(72.7 \%)$ & \\
\hline Reduced but more than a mouthful & $53(53.0 \%)$ & $47(60.3 \%)$ & $6(27.3 \%)$ & \\
\hline
\end{tabular}


Table 1 Demographic and clinical characteristics of enrolled patients ( $n=100)$ (Continued)

\begin{tabular}{|c|c|c|c|c|}
\hline \multirow[t]{2}{*}{ Variable } & \multirow[t]{2}{*}{ Total } & \multicolumn{2}{|l|}{ Hydration } & \multirow[t]{2}{*}{$p$-value } \\
\hline & & $\begin{array}{l}<400 \mathrm{ml} \\
(n=78)\end{array}$ & $\begin{array}{l}\geqq 400 \mathrm{ml} \\
(n=22)\end{array}$ & \\
\hline Bowel obstruction & & & & 0.091 \\
\hline Without & 75 (75.0\%) & 62 (79.5\%) & $13(59.1 \%)$ & \\
\hline With & $25(25.0 \%)$ & $16(20.5 \%)$ & 9 (40.9\%) & \\
\hline Hydration amount & $249.02 \pm 298.50$ & $116.35 \pm 119.03$ & $719.41 \pm 266.29$ & $<0.001$ \\
\hline Transfusion & & & & 0.647 \\
\hline Not used & 93 (93.0\%) & 73 (93.6\%) & 20 (90.9\%) & \\
\hline Used & $7(7.0 \%)$ & $5(6.4 \%)$ & $2(9.1 \%)$ & \\
\hline Antibiotic & & & & 0.088 \\
\hline Not used & $45(45.0 \%)$ & $39(50 \%)$ & $6(27.3 \%)$ & \\
\hline Used & 55 (55.0\%) & 39 (50\%) & $16(72.7 \%)$ & \\
\hline Albumin & & & & 0.334 \\
\hline Not used & $94(94 \%)$ & $72(92.3 \%)$ & $22(100 \%)$ & \\
\hline Used & $6(6.0 \%)$ & $6(7.7 \%)$ & $0(0 \%)$ & \\
\hline Hospitalisation day & $12.04 \pm 6.93$ & $12.55 \pm 6.64$ & $10.23 \pm 7.78$ & 0.063 \\
\hline Total GDS & $13.04 \pm 2.35$ & $13.10 \pm 2.41$ & $12.89 \pm 2.23$ & 0.575 \\
\hline Hospital death & & & & 0.041 \\
\hline No & $32(32.0 \%)$ & $29(37.2 \%)$ & $3(13.6 \%)$ & \\
\hline Yes & 68 (68.0\%) & 49 (62.8\%) & 19 (86.4\%) & \\
\hline
\end{tabular}

Data presented as mean \pm standard deviation (SD) for continuous variables and frequency (percentage, \%) for categorical variables. The $p$-values were calculated using the Wilcoxon rank-sum test for continuous variables and Fisher's exact test for categorical variables

NTUH National Taiwan University Hospital, Chi-Mei Chi-Mei Medical Centre, KMUH Kaohsiung Medical University Hospital, GDS Good Death Scale, GI Gastrointestinal, ECOG Eastern Cooperative Oncology Group performance status

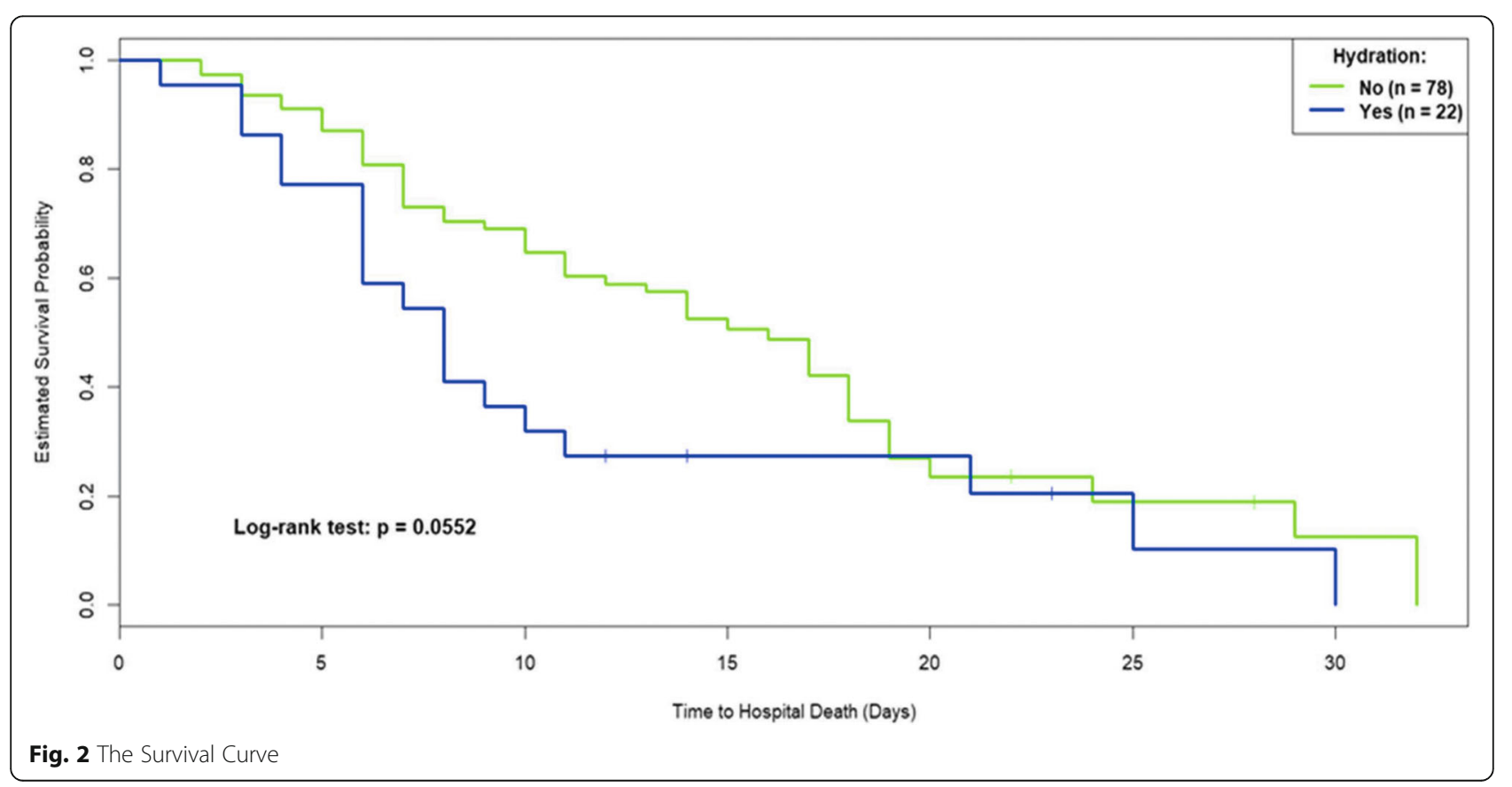


Table 2 Multivariate analyses of the predictors of survival status $(n=68)$

\begin{tabular}{|c|c|c|c|c|c|c|}
\hline Covariates & B & $\begin{array}{l}\text { Standard } \\
\text { Error }\end{array}$ & z-score & $\begin{array}{l}\text { Hazard } \\
\text { Ratio }\end{array}$ & $\begin{array}{l}\text { 95\% Confidence } \\
\text { Interval }\end{array}$ & $p$-value \\
\hline Institution_NTUH & 0.344 & 0.336 & 1.054 & 1.411 & $0.731-2.724$ & 0.305 \\
\hline Age & -0.025 & 0.014 & 3.211 & 0.976 & $0.950-1.002$ & 0.073 \\
\hline ECOG: $3-4$ vs $1-2$ & 2.045 & 1.044 & 3.838 & 7.732 & $0.999-59.839$ & 0.050 \\
\hline Education: $7 \sim 12$ vs $\leqq 6$ & -0.455 & 0.381 & 1.424 & 0.634 & $0.300-1.340$ & 0.233 \\
\hline Education: $>12$ vs $\leqq 6$ & -0.830 & 0.482 & 2.968 & 0.436 & $0.170-1.121$ & 0.085 \\
\hline Religion: Buddhism vs Nullifidian & -0.314 & 0.486 & 0.417 & 0.731 & $0.282-1.893$ & 0.518 \\
\hline Religion: Christian/Catholicism vs Nullifidian & -0.378 & 0.576 & 0.431 & 0.685 & $0.222-2.118$ & 0.512 \\
\hline Religion: Taoism/Taiwanese folk religion vs Nullifidian & 0.208 & 0.477 & 0.189 & 1.231 & $0.483-3.135$ & 0.664 \\
\hline Religion: Unknown vs Nullifidian & 2.287 & 0.986 & 5.383 & 9.844 & $1.426-67.948$ & 0.020 \\
\hline $\begin{array}{l}\text { Oral intake: Less than a mouthful vs Reduced but } \\
\text { more than a mouthful }\end{array}$ & 0.245 & 0.325 & 0.566 & 1.277 & $0.675-2.416$ & 0.452 \\
\hline Hydration: $\geqq 400$ vs $<400$ & 0.353 & 0.368 & 0.923 & 1.424 & $0.692-2.928$ & 0.337 \\
\hline Fatigue admission & 0.544 & 0.242 & 5.049 & 1.722 & $1.072-2.767$ & 0.025 \\
\hline Drowsiness admission & -0.071 & 0.178 & 0.161 & 0.931 & $0.657-1.319$ & 0.688 \\
\hline Dry admission & -0.083 & 0.179 & 0.214 & 0.921 & $0.649-1.307$ & 0.643 \\
\hline Myoclonus admission & -0.200 & 0.373 & 0.287 & 0.819 & $0.394-1.701$ & 0.592 \\
\hline Delirium admission & 0.117 & 0.206 & 0.322 & 1.124 & $0.750-1.684$ & 0.570 \\
\hline Dyspnoea admission & 0.316 & 0.184 & 2.952 & 1.371 & $0.957-1.965$ & 0.086 \\
\hline Oedema admission & 0.384 & 0.162 & 5.602 & 1.469 & $1.068-2.019$ & 0.018 \\
\hline
\end{tabular}

NTUH National Taiwan University Hospital, ECOG Eastern Cooperative Oncology Group performance status, GI Gastro-intestinal

not, whereas previous studies used $1 \mathrm{~L}$ as the cut-off point and the groups who received over $1 \mathrm{~L} \mathrm{AH}$ per day had lower dehydration scores but more fluid retention symptoms. Therefore, giving less than $1 \mathrm{~L}$ or even less than $400 \mathrm{ml} \mathrm{AH}$ per day does not affect the dry mouth or myoclonus symptoms or exacerbate the severity of oedema or dyspnoea in terminally ill cancer patients after 1 week. In previous studies, many symptoms of terminally ill cancer patients had little relationship to $\mathrm{AH}[10,23-25]$, thus routine $\mathrm{AH}$ is not recommended for the treatment of terminally ill cancer patients' symptoms.
This study also found that the administration of $\mathrm{AH}$ to terminally ill cancer patients did not influence survival, similar to previous studies $[6,10]$. According to TorresVigil, African Americans, Latinos and Asian Americans are more likely than non-Hispanic white subjects to view $\mathrm{AH}$ as food or as both food and medicine. Indeed, in a previous study, most terminally ill cancer patients' families regarded $\mathrm{AH}$ as basic care and wanted continuous $\mathrm{AH}$ administration in the hope that the patient's condition would improve [5-7, 16, 17]. Chiu et al. found that most terminally ill cancer patients in the PCU wish to use $\mathrm{ANH}$ and want $\mathrm{AH}$, as they and their families

Table 3 Changes in symptoms between Day 0 and Day $7(n=88)$

\begin{tabular}{|c|c|c|c|c|c|c|c|}
\hline \multirow[t]{2}{*}{ Variable } & \multicolumn{2}{|c|}{ Non-Hydration $(n=72)$} & \multirow[b]{2}{*}{$p$-value } & \multicolumn{2}{|c|}{ With Hydration $(n=16)$} & \multirow[b]{2}{*}{$p$-value } & \multirow[b]{2}{*}{$\begin{array}{l}\text { Between- } \\
\text { groups } p \text {-value }\end{array}$} \\
\hline & Day 0 & Day 7 & & Day 0 & Day 7 & & \\
\hline Fatigue & $2.35 \pm 1.09$ & $2.52 \pm 1.20$ & 0.113 & $2.60 \pm 0.85$ & $3.10 \pm 0.96$ & 0.119 & 0.068 \\
\hline Drowsiness & $1.80 \pm 1.26$ & $2.12 \pm 1.42$ & 0.038 & $1.81 \pm 1.42$ & $2.97 \pm 1.12$ & 0.008 & 0.019 \\
\hline Dry mouth & $1.37 \pm 1.05$ & $1.27 \pm 1.04$ & 0.548 & $2.00 \pm 1.37$ & $1.85 \pm 1.38$ & 0.717 & 0.062 \\
\hline Myoclonus & $0.14 \pm 0.39$ & $0.21 \pm 0.63$ & 0.458 & $0.19 \pm 0.40$ & $0.06 \pm 0.25$ & 0.157 & 0.364 \\
\hline Delirium & $0.47 \pm 0.86$ & $0.56 \pm 0.89$ & 0.182 & $0.63 \pm 0.89$ & $0.63 \pm 0.96$ & 0.861 & 0.690 \\
\hline Dyspnoea & $0.63 \pm 0.80$ & $0.65 \pm 0.83$ & 0.748 & $0.50 \pm 0.73$ & $0.63 \pm 0.81$ & 0.414 & 0.905 \\
\hline Oedema & $0.97 \pm 0.98$ & $0.97 \pm 0.90$ & 0.741 & $1.13 \pm 1.26$ & $1.06 \pm 1.00$ & 0.739 & 0.783 \\
\hline
\end{tabular}

Data are presented as mean \pm SD for continuous variables. The $p$-values were calculated using the Wilcoxon rank-sum test for within-group analyses and MannWhitney $U$ test for between-groups analyses 
Table 4 Multivariate analyses of the predictors of a good death (GDS $\geq 12, n=68$ )

\begin{tabular}{|c|c|c|c|c|c|c|}
\hline Covariates & B & $\begin{array}{l}\text { Standard } \\
\text { Error }\end{array}$ & z-score & $\begin{array}{l}\text { Odds } \\
\text { Ratio }\end{array}$ & $\begin{array}{l}\text { 95\% Confidence } \\
\text { Interval }\end{array}$ & $p$-value \\
\hline \multicolumn{7}{|l|}{ Total score of GDS $\geq 12$} \\
\hline Gl cancer & -1.2134 & 0.7829 & -1.5500 & 0.2972 & $0.064-1.379$ & 0.1212 \\
\hline Genitourinary cancer & -2.4362 & 1.2471 & -1.9535 & 0.0875 & $0.008-1.008$ & 0.0508 \\
\hline Antibiotic use & 1.1199 & 0.7356 & 1.5225 & 3.0644 & $0.725-12.956$ & 0.1279 \\
\hline Albumin use & -2.0167 & 1.4147 & -1.4255 & 0.1331 & $0.008-2.130$ & 0.1540 \\
\hline Religion_Buddhism & 1.8821 & 1.0184 & 1.8481 & 6.5675 & $0.892-48.340$ & 0.0646 \\
\hline Hydration $>86 \mathrm{cc}$ and $\leqq 445 \mathrm{cc}$ & 2.5538 & 0.7824 & 3.2642 & 12.8560 & $2.774-59.575$ & 0.0011 \\
\hline
\end{tabular}

Gl Gastro-intestinal

believed AH could help patients avoid dehydration or starvation or prevent them from starving to death. Also, some patients believe ANH could prolong all patients' life [26]. Huang et al. also found that withdrawing ANH was a difficult decision for families during end-of-life care [27]. In Taiwan, a culture where food intake is strongly related to healing and hope, $\mathrm{AH}$ is regarded as a "lifeline", thus withholding or withdrawal of $\mathrm{AH}$ is often mistakenly regarded as unethical by those who do not understand the role of $\mathrm{AH}$ in terminally ill cancer patients in the stage of actively dying. Many physicians prescribe $\mathrm{AH}$ to allay the fears of family members that the patient might be "starved to death." Once again, our study demonstrated that $\mathrm{AH}$ does not prolong a patient's life, so instead of focusing on the patient's intake, healthcare professionals should explain to families the role of AH during the terminal stages.

Nevertheless, appropriately administering $\mathrm{AH}$ to terminally ill cancer patients could achieve a better quality of dying. In the United States, Cohen et al. found that terminally ill patients and their families believed hydration could bring hope, improve patients' symptoms and enhance QOL [28]. Previous studies which only measure the influence of $\mathrm{AH}$ on QOL found no such remarkable effect [10], however, QOL is not equivalent to the quality of dying, which may be influenced by many other factors than those found in QOL. In our study, appropriate hydration was a predictor of better GDS (GDS $\geqq 12)$. Furthermore, as in many other studies, appropriate hydration may meet the psychological needs and expectations of terminally ill cancer patients and their families [5-7, $16,17]$ by reducing the burden of making difficult decisions and helping both patients and their families to better prepare to face death. Nevertheless, more research is warranted to validate the impact of $\mathrm{AH}$ on the quality of dying of terminally ill cancer patients.

This study was a pilot prospective, multi-centre, observational project and the recruited subjects were from different hospitals in northern and southern Taiwan. While the study may be representative of the national cancer patient population, there were several study limitations.
First, the number of study subjects was small, so future studies should involve more patients to confirm the effect of hydration on terminally ill cancer patients. Second, the imbalance between groups showed that fewer terminally ill cancer patients in Taiwan receive $\mathrm{AH}$, hence there is a risk of sample bias related to the selection of patients referred for palliative care. Third, this study was not blinded, hence, the clinical assessors may have had some preconceived bias. A randomised controlled trial to decrease the bias of statistical analysis and the placebo effect in the future is warranted. Fourth, we did not record the indication of hydration, whether it was mainly under patient/family desire, or physician-led, this should be considered in future studies. Fifth, it was not possible to collect detailed data of median survival from hydration to death in each group as some patients survived and were discharged from PCUs, hence, were not followed up. However, this study only evaluated the hydration effect of survival status in the hospital, not the whole survival condition. In future, patients could be followed up until death, even if they are discharged. Finally, the two groups of patients were not comparable in terms of the characteristics of age, education and religion. Nevertheless, we performed regression analysis to adjust for these differences. This is a pilot study conducted in Asia, and a large-scale, cross-cultural, multicentre study is ongoing based on the results of this pilot study.

\section{Conclusions}

For terminally ill cancer patients in PCU, AH over 400 $\mathrm{mL}$ might not prolong survival nor significantly improve the dehydration symptoms, but appropriate AH may improve the quality of dying. Hydration remains an ethical dilemma, especially in the Asian context. Communication with patients and their families is recommended regarding the benefit and adverse effects of $\mathrm{AH}$, as this may help better prepare them for the final stage of life and achieve a good death. In the future, a large-scale randomised-controlled study of the impact of $\mathrm{AH}$ on the quality of dying is warranted. 


\section{Abbreviations}

QOL: Quality of life; $\mathrm{AH}$ : Artificial hydration; $\mathrm{ANH}$ : Artificial nutrition and hydration; PCU: Palliative care units; GDS: Good death scale; ECOG: Eastern Cooperative Oncology Group.; IPOS: Integrated Palliative care Outcome Scale; MDAS: Memorial Delirium Assessment Scale; HR: Hazard ratio; Cl: Confidence Interval; OR: Odds ratio

\section{Acknowledgements}

The authors would like to thank the patients and the staff of the palliative care units of the three tertiary hospitals in Taiwan (National Taiwan University Hospital, Chi-Mei Medical Centre and Kaohsiung Medical University Hospital).

\section{Authors' contributions}

CYW: collected the study data and was a major contributor in writing the manuscript. PJC: contributed to the trial design, protocol development, collection of the study data and manuscript review. TLH: assisted the organisation and analysis of the study data. WYL: contributed to the trial design and protocol development. SYC: contributed to the trial design, protocol development, collection and analysis of the study data, and manuscript review. All authors read and approved the final manuscript

\section{Funding}

This research was supported by Ministry of Science and Technology in Taiwan (105-2314-B-002-172). The funding bodies played no role in the design of the study and collection, analysis, and interpretation of data and in writing the manuscript.

\section{Availability of data and materials}

The datasets used and analysed in the current study are available from the corresponding author on reasonable request.

\section{Ethics approval and consent to participate}

This study was approved by Institutional Review Boards of all three hospitals (National Taiwan University Hospital, Chi-Mei Medical Centre and Kaohsiung Medical University Hospital). If the patients met the inclusion criteria, the researchers explained the study purpose and protocol to the patients and their families, and they provided written informed consent to participate in the study.

\section{Consent for publication}

Not applicable.

\section{Competing interests}

The authors declare that they have no competing interests.

\section{Author details}

'Department of Family Medicine, Kaohsiung Medical University Hospital, Kaohsiung Medical University, Kaohsiung, Taiwan. ${ }^{2}$ Division of Geriatrics and Gerontology, Kaohsiung Medical University Hospital, Kaohsiung Medical University, Kaohsiung, Taiwan. ${ }^{3}$ Marie Curie Palliative Care Research Department, Division of Psychiatry, University College London, London, UK ${ }^{4}$ Department of Family Medicine, College of Medicine and University Hospital, National Taiwan University, 7 Chung-Shan South Road, Taipei 100, Taiwan. ${ }^{5}$ Department of Family Medicine, China Medical University Hospital, Taichung, Taiwan. ${ }^{6}$ School of Medicine, College of Medicine, China Medical University, Taichung, Taiwan.

Received: 14 July 2020 Accepted: 3 January 2021

Published online: 12 January 2021

\section{References}

1. O'Mahony S, Blank AE, Zallman L, Selwyn PA. The benefits of a hospitalbased inpatient palliative care consultation service: preliminary outcome data. J Palliat Med. 2005;8(5):1033-9.

2. Temel JS, Greer JA, Muzikansky A, Gallagher ER, Admane S, Jackson VA, Dahlin CM, Blinderman CD, Jacobsen J, Pirl WF. Early palliative care for patients with metastatic non-small-cell lung cancer. N Engl J Med. 2010; 363(8):733-42.

3. Cheng S-Y, Dy S, Fang P-H, Chen C-Y, Chiu T-Y. Evaluation of inpatient multidisciplinary palliative care unit on terminally ill cancer patients from providers' perspectives: a propensity score analysis. Jpn J Clin Oncol. 2012 43(2):161-9.

4. Grosvenor M, Bulcavage L, Chlebowski RT. Symptoms potentially influencing weight loss in a cancer population. Correlations with primary site, nutritional status, and chemotherapy administration. Cancer. 1989:63(2):330-4.

5. Chiu TY, Hu WY, Chen CY. Prevalence and severity of symptoms in terminal cancer patients: a study in Taiwan. Support Care Cancer. 2000; 8(4):311-3.

6. Chiu T-Y, Hu W-Y, Chuang R-B, Chen C-Y. Nutrition and hydration for terminal cancer patients in Taiwan. Support Care Cancer. 2002;10(8):630-6.

7. Mercadante S, Ferrera P, Girelli D, Casuccio A. Patients' and relatives' perceptions about intravenous and subcutaneous hydration. J Pain Symptom Manag. 2005;30(4):354-8.

8. Ganzini L. Artificial nutrition and hydration at the end of life: ethics and evidence. Palliat Support Care. 2006;4(2):135-43.

9. Geppert CM, Andrews MR, Druyan ME. Ethical issues in artificial nutrition and hydration: a review. J Parenter Enter Nutr. 2010;34(1):79-88.

10. Bruera E, Hui D, Dalal S, Torres-Vigil I, Trumble J, Roosth J, Krauter S, Strickland C, Unger K, Palmer JL. Parenteral hydration in patients with advanced cancer: a multicenter, double-blind, placebo-controlled randomized trial. J Clin Oncol. 2013;31(1):111.

11. Morita $T$, Tei $Y$, Inoue S. Agitated terminal delirium and association with partial opioid substitution and hydration. J Palliat Med. 2003;6(4): 557-63.

12. Morita T, Shima Y, Miyashita M, Kimura R, Adachi I, Group JPOS. Physicianand nurse-reported effects of intravenous hydration therapy on symptoms of terminally ill patients with cancer. J Palliat Med. 2004;7(5):683-93.

13. Morita T, Hyodo I, Yoshimi T, Ikenaga M, Tamura Y, Yoshizawa A, Shimada A, Akechi T, Miyashita M, Adachi I. Association between hydration volume and symptoms in terminally ill cancer patients with abdominal malignancies. Ann Oncol. 2005:16(4):640-7.

14. Morita T, Hyodo I, Yoshimi T, Ikenaga M, Tamura Y, Yoshizawa A, Shimada A, Akechi T, Miyashita M, Adachi I. Artificial hydration therapy, laboratory findings, and fluid balance in terminally ill patients with abdominal malignancies. J Pain Symptom Manag. 2006;31(2): $130-9$.

15. Morita T, Bito S, Koyama H, Uchitomi Y, Adachi I. Development of a national clinical guideline for artificial hydration therapy for terminally ill patients with cancer. J Palliat Med. 2007;10(3):770-80.

16. Morita T, Tsunoda J, Inoue S, Chihara S. Perceptions and decision-making on rehydration of terminally ill cancer patients and family members. Am J Hosp Palliat Med. 1999:16(3):509-16.

17. Morita T, Miyashita M, Shibagaki M, Hirai K, Ashiya T, Ishihara T, Matsubara T, Miyoshi I, Nakaho T, Nakashima N. Knowledge and beliefs about end-of-life care and the effects of specialized palliative care: a population-based survey in Japan. J Pain Symptom Manag. 2006;31(4):306-16.

18. Torres-Vigil I, Cohen MZ, de la Rosa A, Cárdenas-Turanzas M, Burbach BE, Tarleton KW, Shen W-M, Bruera E. Food or medicine: ethnic variations in perceptions of advanced cancer patients and their caregivers regarding artificial hydration during the last weeks of life. BMJ Support Palliat Care. 2012; bmjspcare-2012-000205.

19. Cheng S-Y, Hu W-Y, Liu W-J, Yao C-A, Chen C-Y, Chiu T-Y. Good death study of elderly patients with terminal cancer in Taiwan. Palliat Med. 2008;22(5): 626-32.

20. Yao C-A, Hu W-Y, Lai Y-F, Cheng S-Y, Chen C-Y, Chiu T-Y. Does dying at home influence the good death of terminal cancer patients? J Pain Symptom Manag. 2007;34(5):497-504.

21. Leung K-K, Tsai J-S, Cheng S-Y, Liu W-J, Chiu T-Y, Wu C-H, Chen C-Y. Can a good death and quality of life be achieved for patients with terminal cancer in a palliative care unit? J Palliat Med. 2010;13(12):1433-8.

22. Nakajima N, Hata Y, Kusumuto K. A clinical study on the influence of hydration volume on the signs of terminally ill cancer patients with abdominal malignancies. J Palliat Med. 2013;16(2):185-9.

23. Ellershaw JE, Sutcliffe JM, Saunders CM. Dehydration and the dying patient. J Pain Symptom Manag. 1995:10(3):192-7.

24. McCann RM, Hall WJ, Groth-Juncker A. Comfort care for terminally iii patients: the appropriate use of nutrition and hydration. Jama. 1994;272(16): 1263-6.

25. Burge Fl. Dehydration symptoms of palliative care cancer patients. J Pain Symptom Manag. 1993;8(7):454-64. 
26. Chiu T-Y, Hu W-Y, Chuang R-B, Cheng Y-R, Chen C-Y, Wakai S. Terminal cancer patients' wishes and influencing factors toward the provision of artificial nutrition and hydration in Taiwan. J Pain Symptom Manag. 2004; 27(3):206-14.

27. Huang HL, Chiu TY, Lee LT, Yao CA, Chen CY, Hu WY. Family experience with difficult decisions in end-of-life care. Psycho-Oncology. 2012;21(7):785-91.

28. Cohen MZ, Torres-Vigil I, Burbach BE, De La Rosa A, Bruera E. The meaning of parenteral hydration to family caregivers and patients with advanced cancer receiving hospice care. J Pain Symptom Manag. 2012;43(5):855-65.

\section{Publisher's Note}

Springer Nature remains neutral with regard to jurisdictional claims in published maps and institutional affiliations.

Ready to submit your research? Choose BMC and benefit from:

- fast, convenient online submission

- thorough peer review by experienced researchers in your field

- rapid publication on acceptance

- support for research data, including large and complex data types

- gold Open Access which fosters wider collaboration and increased citations

- maximum visibility for your research: over $100 \mathrm{M}$ website views per year

At $\mathrm{BMC}$, research is always in progress.

Learn more biomedcentral.com/submissions 